\title{
Expansion of the All-Sky Radiance Assimilation to ATMS at NCEP
}

\author{
YAnqiu Zhu, George Gayno, R. James Purser, Xiujuan Su, and Runhua Yang \\ I.M. Systems Group, Inc., and NOAA/NCEP/Environmental Modeling Center, College Park, Maryland
}

(Manuscript received 27 June 2018, in final form 31 January 2019)

\begin{abstract}
Since the implementation of all-sky radiance assimilation of the Advanced Microwave Sounding Unit-A (AMSU-A) in the operational hybrid 4D ensemble-variational Global Forecast System at NCEP in 2016, the all-sky approach has been tested to expand to the radiances of Advanced Technology Microwave Sounder (ATMS) in the Gridpoint Statistical Interpolation analysis system (GSI). Following the all-sky framework implemented for the AMSU-A radiances, ATMS radiance assimilation adopts similar procedures in quality control, bias correction, and model of observation error. Efforts have been focused on special considerations that are necessary because of the unique features of the ATMS radiances and water vapor channels, including surface properties based on fields of view size and shape, and taking care of large departures from the first guess $(\mathrm{OmF})$ along coastlines and radiances affected by strong scattering. More importantly, it is shown that this work makes microwave radiance OmFs become more consistent among different sensors, and provides indications of the deficiencies in quality control procedures of the original ATMS and Microwave Humidity Sounder (MHS) clear-sky radiance assimilation. While the generalized tracer effect is noticed, the overall impact on the forecast skill is neutral. This work is included in the upcoming operational implementation in 2019.
\end{abstract}

\section{Introduction}

In the past decade, with the advances of forecast models and the improvement of radiative transfer models (Bauer et al. 2006; Liu and Weng 2006; Ding et al. 2011), Numerical weather prediction (NWP) centers have made steady progress toward utilizing cloudy radiances in addition to radiance observations in clear sky. The European Centre for Medium-Range Weather Forecasts (ECMWF) realized direct all-sky radiance assimilation for the Special Sensor Microwave Imager (SSM/I) and the Advanced Microwave Scanning Radiometer for Earth Observing System (AMSR-E) in 2009 (Bauer et al. 2010; Geer et al. 2012). Comprehensive studies have also been conducted in other NWP centers such as the Met Office, Japan Meteorological Agency, Météo-France, and Deutscher Wetterdienst (Okamoto 2013; Migliorini et al. 2017; Martinet et al. 2013). In the Gridpoint Statistical Interpolation analysis system (GSI; Derber et al. 1991; Parrish and Derber 1992; Wu et al. 2002) at the National Centers for Environmental Prediction (NCEP), the capability of all-sky radiance assimilation has been

\footnotetext{
Corresponding author: Yanqiu Zhu, yanqiu.zhu@noaa.gov
}

developed, and the assimilation of cloudy radiances from the Advanced Microwave Sounding Unit-A (AMSU-A) microwave radiometer for ocean fields of view (FOV) became operational in the Global Forecast System (GFS) in 2016 (Zhu et al. 2016) as the GFS was upgraded to the 4D hybrid ensemble-variational (EnVar) system. The assimilation of cloudy AMSU-A radiances in the GFS improves the temperature and relative humidity as well as reducing a known positive bias of stratus amount. Since then, efforts have been made in the expansion of the all-sky approach to the radiances from other sensors in the GSI.

In addition to the AMSU-A, radiances from the Advanced Technology Microwave Sounder (ATMS) on board Suomi National Polar-Orbiting Partnership $(S N P P)$ constitute one of the major datasets of microwave radiances assimilated in the GSI analysis system. Our work describes in detail the expansion of the all-sky approach to ATMS radiances. Like the operational AMSU-A radiance assimilation, normalized cloud water is used as the cloud control variable in this study, symmetric observation error (Geer and Bauer 2011) is assigned to ATMS radiances as a function of cloud amount, situation-dependent observation error inflation (SDOEI) is applied to improve the system's 
performance, and the same bias correction approach as for AMSU-A (Zhu et al. 2014b) is adopted for ATMS radiances. The focus of the all-sky expansion to ATMS is primarily on the unique features of the ATMS radiances, for example, the handling of large observation departures from the first guess $(\mathrm{OmF})$ along coastlines, and the inclusion of water vapor channels. The corresponding procedures related to quality control are discussed.

As a growing quantity of observations is assimilated, it is necessary to assess the compatibility of observational information from different sources. Observations that are not in agreement with each other will have negative impacts on the analysis and system performance. The observation inconsistency may come from the instrument and calibration errors, but it may also arise from the various procedures and processing algorithms in the data assimilation systems, such as quality control or bias correction. In this study, data inconsistencies from the latter case are examined in section 4 . In the current operational GFS system, only AMSU-A radiances are assimilated using the all-sky approach; other microwave radiances from ATMS and the Microwave Humidity Sounder (MHS) are assimilated using the clear-sky approach, but with slightly different flavors. As we move toward using the all-sky ATMS radiance assimilation, the impact on the OmF pattern and the data consistency among various sensors is being investigated. With the improved data consistency demonstrated in section 4, the all-sky ATMS radiance assimilation is currently being included in the parallel experiment for the upcoming operational implementation in 2019.

This paper is organized as follows: A brief description of the GSI analysis system is provided in section 2. Expansion of the all-sky approach to ATMS and special considerations for ATMS radiances are presented in section 3 , followed by the discussion of data consistency among AMSU-A, ATMS, and MHS in section 4. The impacts of going from the clear-sky to all-sky approach for the ATMS radiances on the forecast skills are shown in section 5 . Last, current work and future plans are discussed in section 6. For convenience, a list of acronyms is provided in Table 1.

\section{The operational GSI analysis system}

The GSI analysis system combines the atmospheric states generated from the forecast model and the observations to produce better estimates of atmospheric states for the initial conditions of the forecast model,
TABLE 1. A list of acronyms or abbreviations.

\begin{tabular}{ll}
\hline \hline \multicolumn{1}{c}{ Acronym } & \multicolumn{1}{c}{ Expansion } \\
\hline AAPP & ATOVS and AVHRR Preprocessing Package \\
AMSR-E & $\begin{array}{l}\text { Advanced Microwave Scanning Radiometer for } \\
\text { Earth Observing System }\end{array}$ \\
AMSU-A & Advanced Microwave Sounding Unit-A \\
ATMS & Advanced Technology Microwave Sounder \\
ATOVS & Advanced TIROS Operational Vertical Sounder \\
AVHRR & Advanced Very High Resolution Radiometer \\
CLW & Cloud liquid water \\
CRTM & Community Radiative Transfer Model \\
CTL & Control experiment \\
ECMWF & European Centre for Medium-Range Weather \\
& Forecasts \\
EnVar & Ensemble-variational \\
EUMETSAT & European Organisation for the Exploitation of \\
& $\quad$ Meteorological Satellites \\
FFT & Fast Fourier transform \\
FOV & Field of view \\
FV3 & Finite-Volume Cubed-Sphere Dynamical Core \\
GFS & Global Forecast System \\
GPS & Global positioning system \\
GSI & Gridpoint Statistical Interpolation analysis system \\
MHS & Microwave Humidity Sounder \\
NCEP & National Centers for Environmental Prediction \\
NMC & National Meteorological Center \\
NWP & Numerical weather prediction \\
OmF & Observation minus forecast \\
SAF & Satellite Application Facility \\
SDOEI & Situation-dependent observation error inflation \\
SI & Scattering index \\
SSM/I & Special Sensor Microwave Imager \\
SSMIS & Special Sensor Microwave Imager/Sounder \\
STDV & Standard deviation \\
$T_{B}$ & Brightness temperature \\
VQC & Variational quality control \\
\hline &
\end{tabular}

where the observational information is propagated through the background error covariance. In the current operational 4D hybrid EnVar GFS system (Wang and Lei 2014; Kleist and Ide 2015), the background error covariance is composed of the static term and a part generated from the ensemble forecasts, with $87.5 \%$ weight given to the ensemble part. The ensemble part is achieved through a combination of ensemble data assimilation using the ensemble serial square root filter (Whitaker and Hamill 2002), multiplicative inflation by relaxation to the prior spread, and inclusion of stochastic parameterization schemes. The static term is generated by the National Meteorological Center (NMC) method (Parrish and Derber 1992). The NMC method estimates the forecast errors using the rescaled differences between 24 - and 48 -h forecasts but valid at the same time.

Conventional, global positioning system (GPS) radio occultation, and satellite wind and radiance data are assimilated in the system. Except for microwave 
AMSU-A radiances, all other radiance observations, including two other microwave radiances, ATMS and MHS, are assimilated in the clear-sky approach, where cloud information is not provided to the Community Radiative Transfer Model (CRTM), version 2.2.3, in the calculation of simulated brightness temperature $T_{B}$. The GFS system started to assimilate the ATMS radiances in the clear-sky approach in 2012 (Collard et al. 2012). ATMS has 22 similar channels to AMSU-A/ MHS and most of the AMSU-A/MHS processing is directly applied to ATMS radiances. Since ATMS has different field of view sizes and separations, the ATOVS and AVHRR Preprocessing Package [AAPP, NWP Satellite Application Facility (SAF)/EUMETSAT] with remapping and spatial averaging is applied to the AMSU-A-like ATMS channels (channels 1-16) to a common FOV of $3.3^{\circ}$ (Collard et al. 2012). In the GSI, ATMS and MHS radiances are assimilated slightly differently; while MHS radiances affected by clouds and precipitation are excluded in the quality control procedure, ATMS radiances in clear-sky and affected by optically thin clouds are used, with an attempt to remove the cloud effect by a cloud liquid water bias predictor.

The AMSU-A radiances are assimilated in the all-sky approach (Zhu et al. 2016), where clouds are taken into account in the calculation of simulated $T_{B}$ in the CRTM. In the current operational GFS system with Zhao and Carr (1997) microphysics, cloud water (CW, the sum of the cloud liquid water and cloud ice) is the cloud prognostic variable in the model and provided to the GSI, but other information on precipitation, snow, and graupel from the forecast model is not available for use in the GSI. Therefore, only radiances affected by nonprecipitating clouds and clear-sky radiances are used. Normalized cloud water is used as the cloud control variable. The cloud background error standard deviation in the static term is specified as $5 \%$ of the cloud water first guess from the deterministic forecast, and both horizontal and vertical correlation lengths are set to be half of those for the relative humidity. The cross-covariances between cloud water and other control variables are not specified in the static term, but are instead provided by the 80 ensemble members. Like in the forecast model, $\mathrm{CW}$ is decomposed into cloud liquid water and cloud ice state variables based on temperature $T$, with $f \times \mathrm{CW}$ being cloud ice and the rest cloud liquid water, where

$$
f= \begin{cases}0 & (\tilde{f}<0) \\ \tilde{f} & (0 \leq \tilde{f} \leq 1) \\ 1 & (\tilde{f}>1)\end{cases}
$$

and $\tilde{f}=0.05 \times(273.15-T)$. Cloud liquid water and cloud ice are required by the CRTM in the simulated radiance calculation. The tangent linear and adjoint of this decomposition operator are also employed for increment conversion between cloud water control variable and cloud liquid water and cloud ice state variables at each iteration of the inner loop. The radiance data information is mapped onto not only the temperature and moisture fields as in the clear-sky approach, but also cloud fields via the brightness temperature Jacobians with respect to cloud liquid water and cloud ice. A cloud seed of $1.001 \times 10^{-6} \mathrm{~kg} \mathrm{~m}^{-2}$ is provided to the radiative transfer model as input to calculate cloud Jacobians for cloud-free layers.

The operational GSI analysis system employs an incremental method with two outer loops. At each outer loop, the iterative process of the inner loop stops when the convergence criterion is satisfied, or until the maximum 50 inner iterations for the first outer loop and 150 for the second outer loop are reached. In the inner loop, linearized observation operators are used, and control variables are updated at the end of each outer loop and provided to the next outer loop as the new background fields. Because of the nonlinearity of the radiance observation operator for cloudy radiances, all-sky radiance assimilation benefits from the configuration of multiple outer loops as demonstrated in Bauer et al. (2010).

\section{Expansion of the all-sky radiance assimilation approach to ATMS}

ATMS has 22 channels and combines most of the channels from AMSU-A and MHS, providing measurements of atmospheric temperature and moisture profiles (Table 2). ATMS is different from AMSU-A and MHS in beamwidth, number of fields of view, and scan swath width. Some channels also have different frequency/polarization. Cloud liquid water absorption is significant for lower-peaking channels, such as channels 1 and 2, but scattering effects become more important for channels 16 and 17 and for water vapor channels. Since the all-sky radiance assimilation framework has already been constructed in the previous all-sky AMSUA radiance assimilation work, the expansion of the allsky approach to ATMS radiances has taken a similar approach. Extra efforts on procedures of quality control, observation error assignment, and bias correction are presented in the following subsections. With the MHSlike channels, this work also introduces water vapor channels to the all-sky approach.

\section{a. Quality control}

Unlike AMSU-A, ATMS has varied beamwidths, that is, $5.2^{\circ}$ for channels 1 and $2,2.2^{\circ}$ for channels $3-16$, and 
TABLE 2. Characteristics of AMSUA, MHS, and ATMS instruments. Polarization: V is vertical and H is horizontal.

\begin{tabular}{|c|c|c|c|}
\hline Channel & $\mathrm{GHz}$ and polarization & Channel & $\mathrm{GHz}$ and polarization \\
\hline AMSU-A 1 & $23.8, \mathrm{~V}$ & ATMS 1 & $23.8, \mathrm{~V}$ \\
\hline AMSU-A 2 & $31.399, \mathrm{~V}$ & ATMS 2 & $31.4, \mathrm{~V}$ \\
\hline \multirow[t]{2}{*}{ AMSU-A 3} & $50.299, \mathrm{~V}$ & ATMS 3 & $50.3, \mathrm{H}$ \\
\hline & & ATMS 4 & $51.76, \mathrm{H}$ \\
\hline AMSU-A 4 & $52.8, \mathrm{~V}$ & ATMS 5 & $52.8, \mathrm{H}$ \\
\hline AMSU-A 5 & $53.596 \pm 0.115, \mathrm{H}$ & ATMS 6 & $53.596 \pm 0.115, \mathrm{H}$ \\
\hline AMSU-A 6 & $54.4, \mathrm{H}$ & ATMS 7 & $54.4, \mathrm{H}$ \\
\hline AMSU-A 7 & $54.94, \mathrm{~V}$ & ATMS 8 & $54.94, \mathrm{H}$ \\
\hline AMSU-A 8 & $55.5, \mathrm{H}$ & ATMS 9 & $55.5, \mathrm{H}$ \\
\hline AMSU-A 9 & $57.29, \mathrm{H}$ & ATMS 10 & $57.29, \mathrm{H}$ \\
\hline AMSU-A 10 & $57.29 \pm 0.217, \mathrm{H}$ & ATMS 11 & $57.29 \pm 0.217, \mathrm{H}$ \\
\hline AMSU-A 11 & $57.29 \pm 0.3222 \pm 0.048, \mathrm{H}$ & ATMS 12 & $57.29 \pm 0.3222 \pm 0.048, \mathrm{H}$ \\
\hline AMSU-A 12 & $57.29 \pm 0.3222 \pm 0.022, \mathrm{H}$ & ATMS 13 & $57.29 \pm 0.3222 \pm 0.022, \mathrm{H}$ \\
\hline AMSU-A 13 & $57.29 \pm 0.3222 \pm 0.010, \mathrm{H}$ & ATMS 14 & $57.29 \pm 0.3222 \pm 0.010, \mathrm{H}$ \\
\hline AMSU-A 14 & $57.29 \pm 0.3222 \pm 0.0045, \mathrm{H}$ & ATMS 15 & $57.29 \pm 0.3222 \pm 0.0045, \mathrm{H}$ \\
\hline AMSU-A 15 & $89.0, \mathrm{~V}$ & & \\
\hline MHS 1 & $89.0, \mathrm{~V}$ & ATMS 16 & $88.2, \mathrm{~V}$ \\
\hline \multirow[t]{3}{*}{ MHS 2} & 157.0, V & ATMS 17 & $165.5, \mathrm{H}$ \\
\hline & & ATMS 18 & $183.31 \pm 7, \mathrm{H}$ \\
\hline & & ATMS 19 & $183.31 \pm 4.5, \mathrm{H}$ \\
\hline MHS 4 & $183.31 \pm 3, \mathrm{H}$ & ATMS 20 & $183.31 \pm 3, \mathrm{H}$ \\
\hline MHS 5 & 190.31, V & ATMS 21 & $183.31 \pm 1.8, \mathrm{H}$ \\
\hline MHS 3 & $181.31 \pm 1, \mathrm{H}$ & ATMS 22 & $183.31 \pm 1, \mathrm{H}$ \\
\hline
\end{tabular}

$1.1^{\circ}$ for channels $17-22$. In the current operational clearsky approach for ATMS radiance assimilation, the AAPP remapping and spatial averaging package is applied to only channels 1-16 to convert the beamwidths to $3 \cdot 3^{\circ}$. However, a common beamwidth for all channels is necessary, as the quality control procedures of cloud and scattering detection are based on the assumption that all channels have the same beamwidth. For example, the estimate of cloud liquid water $\left(\mathrm{CLW}\right.$, in units of $\left.\mathrm{kg} \mathrm{m}^{-2}\right)$ at each observation location is obtained with the retrieval formula of Grody et al. (2001) and Weng et al. (2003) using the brightness temperatures $T_{B}(1)$ and $T_{B}(2)$ of channels 1 and 2 ,

$$
\begin{aligned}
\mathrm{CLW}= & \cos \theta \times\left\{c_{0}+c_{1} \ln \left[285-T_{B}(1)\right]\right. \\
& \left.+c_{2} \ln \left[285-T_{B}(2)\right]\right\},
\end{aligned}
$$

where $c_{0}$ (in unit of $\mathrm{kg} \mathrm{m}^{-2}$ ) is calculated as $c_{0}=8.240-$ $(2.622-1.846 \times \cos \theta) \times \cos \theta, \quad c_{1}=0.754 \mathrm{~kg} \mathrm{~m}^{-2}, c_{2}=$ $-2.265 \mathrm{~kg} \mathrm{~m}^{-2}, \theta$ is the zenith angle, and the sea surface temperature is approximated to be $285 \mathrm{~K}$ in the retrieval formula. This CLW estimate has been applied to all channels for cloud detection quality control in the clear-sky approach. Hence, in this allsky ATMS study, the AAPP remapping and spatial averaging package is applied to all channels of ATMS radiances to obtain a common beamwidth of $3.3^{\circ}$, facilitating the calculation of FOV and cloud amount, and quality control procedures. The CLW estimate is also used in symmetric observation error assignment and bias correction. The spatial averaging improves the root-mean-square errors (RMSEs) of radiance OmFs for channels 17-22 when converting to a coarser resolution.

As in the operational GFS system, ATMS radiances over ice, snow, and mixed surfaces are not used in this study, and only radiances of clear sky are assimilated over land with emissivity sensitivity check, where radiances of surface-sensitive channels are excluded if any of the ratios between OmF and emissivity Jacobian for channels $1,2,3$, and 16 is larger than their thresholds. The thresholds are $0.02,0.015,0.035$, and 0.015 for channels $1,2,3$, and 16 , respectively. In this study, an interchannel quality control is added. For each profile, if any one of the cloud/precipitation-affected channels fails the quality control criterion, then all of these channels are not used.

1) QuALITY CONTROL ON RADIANCES WITH LARGE OMFS AROUND COASTLINES AND CRYOSPHERE BOUNDARIES

Unlike operational all-sky AMSU-A radiance assimilation where radiances over mixed surface type locations are assimilated, it has been noticed in this study that ATMS radiances have large OmFs around coastlines and cryosphere boundaries. In the operational GFS, since a very tight gross error check has 

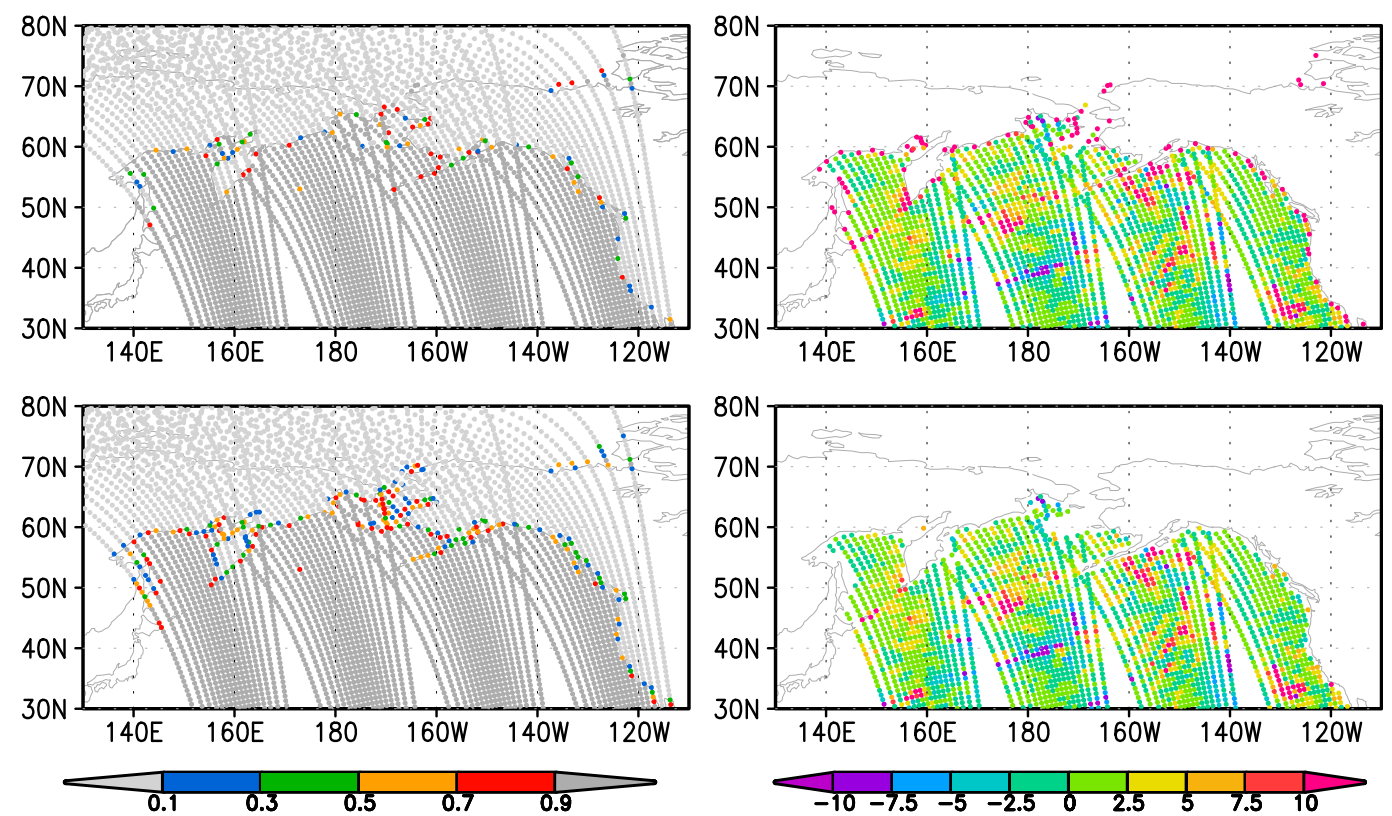

FIG. 1. (Left) Water surface percentage (\%) when surface type is calculated by interpolation using (top) the four nearest model surface grid points and (bottom) with FOV calculation where the relative antenna power at the FOV edge decreases to $1 \%$ of the maximum at the center. Colored points are the mixed surface type locations. (Right) The corresponding brightness temperature OmFs over water for ATMS channel 2 (the unit is K).

been applied to ATMS radiances, these radiance data are not a problem for the clear-sky ATMS radiance assimilation. However, in the all-sky ATMS radiance assimilation, since radiances affected by clouds are usually associated with large OmFs, the gross error check is significantly relaxed (e.g., the gross error check threshold is relaxed from 2.0 to $10.0 \mathrm{~K}$ for channel 17) to allow these cloudy radiances get into the data assimilation system. As the magnitude of the large OmFs around coastlines and cryosphere boundaries may be comparable to those of cloudy radiances, it is necessary to exclude these large OmFs in the quality control to avoid undesired large analysis increments.

In the operational clear-sky approach to ATMS radiance assimilation, surface properties (including land/ sea fraction) at observation locations are calculated as interpolations using the four nearest model surface grid points. This is not appropriate given the resolution of the 4D EnVar system and the large size of the FOV. The resolution of the current operational GFS 4D EnVar system is T1534/T574, that is, $13 \mathrm{~km}$ for the deterministic forecast model and $39 \mathrm{~km}$ for analysis and ensemble. The resolution of analysis and ensemble will have been increased to $25 \mathrm{~km}$ starting 2019. Therefore, the capability of modeling surface properties based on the FOV size and shape is exercised for the all-sky
ATMS radiances. In the GSI, the shape of the FOV is approximated by a 30-sided polygon, and the surface variables are calculated as spatial averages weighted by the antenna pattern within the FOV. An example at 0000 UTC 15 May 2015 is provided in Fig. 1. The left column shows the water surface percentage (\%) using the two methods, and the right column shows the corresponding OmFs over water for ATMS channel 2. As expected, more observation locations are marked as mixed surface type around the coastline after using the FOV calculation. However, ATMS radiances with large OmFs are still found around the coastline, though the radiance OmF RMSE is reduced. The difference is that these large OmFs are associated with the mixed surface type locations when the FOV calculation is activated, while they are associated with both water and mixed surface type locations when nearest-gridpoint interpolation is used. As a result, it is seen that these large OmFs are removed effectively from the system when the ATMS radiances over mixed surface type locations are excluded in the quality control.

\section{2) QUALITY CONTROL ON RADIANCES AFFECTED BY STRONG SCATTERING}

With the introduction of MHS-like channels into the all-sky framework, the scattering effect increases because 
of the higher frequencies. In principle, the CRTM can simulate scattering from hydrometeors in the microwave region. However, as mentioned earlier, in the current operational GFS system, while cloud water is the model prognostic variable and is provided to the GSI, snow and precipitation information from the model is not available in the first guess. In the CRTM, nonprecipitation clouds (cloud liquid water and cloud ice) are assumed to be very small particles in comparison to microwave wavelengths, thus the scattering is not considered by design when there is no snow, graupel and precipitation. Considering the consistency between the observations and the simulated radiances from the CRTM, a new quality control procedure is conducted in this subsection to exclude those radiances that are affected by strong scattering. Bennartz et al. (2002) isolated the scattering signal over land using a scattering index given by the difference between a low-frequency channel and a high-frequency channel brightness temperature. Baordo and Geer (2015) employed the average of such observed and simulated scattering index in the symmetric observation error model and quality control for SSMIS radiance data over land. In this study, in order to investigate the scattering effect over ocean FOVs, we first define the impact of clouds on radiance data, that is, cloud effect cldef, as the following:

$$
\text { cldef }=T_{B}^{\mathrm{cld}}-T_{B}^{\mathrm{clr}} .
$$

It is calculated as the difference between the brightness temperature with and without hydrometeor information taken into account, that is, $T_{B}^{\text {cld }}$ and $T_{B}^{\text {clr }}$. The simulated brightness temperature of clear-sky is used as $T_{B}^{\mathrm{clr}}$. Although $T_{B}^{\mathrm{clr}}$ may involve model error, the accuracy of simulated clear-sky brightness temperature is generally acceptable for the purpose of calculating cldef. $T_{B}^{\text {cld }}$ can be defined by the radiance observations or the simulated brightness temperatures calculated in the CRTM with atmospheric state and clouds taken from the first guess, thus the cloud effect is referred to observed and simulated cloud effect, respectively. For our quality control purpose, since the radiance observations respond to the true representation of the cloud, the observed cloud effect is used in preference to the simulated cloud effect where the representativeness errors of the CRTM and the NWP model, and lack of precipitation and snow information in the first guess, can cause large errors of $T_{B}^{\text {cld }}$ preventing an accurate description of the cloudy scenes.

Since ATMS channels $17(165.5 \mathrm{GHz})$ and 16 $(88.2 \mathrm{GHz})$ have different sensitivities to hydrometeors, and channel 17 is more sensitive to larger hydrometeor
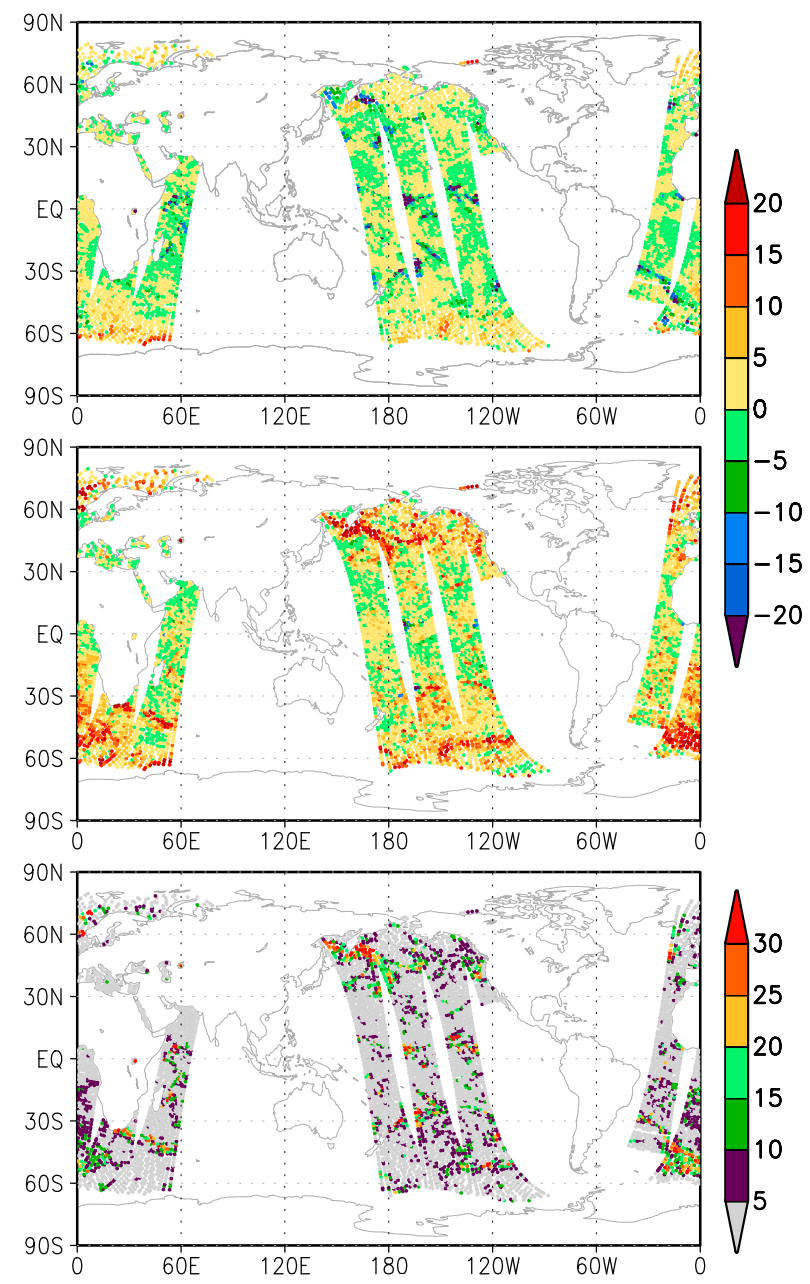

FIG. 2. Cloud effect on ATMS channels (top) 17 and (middle) 16 and (bottom) scattering index at 0000 UTC 1 Jun 2015. The unit is $\mathrm{K}$.

particles like snow, graupel and hail because of shorter wavelength than channel 16 (top and middle panels of Fig. 2), the difference of cloud effects of these two channels is used to define a scattering index (SI) measurement (bottom panel of Fig. 2), that is,

$$
\mathrm{SI}=\operatorname{cldef}(\operatorname{ch} 16)-\operatorname{cldef}(\operatorname{ch} 17) \text {. }
$$

It is shown in Fig. 2 that strong scattering is observed in locations of large cloud condensate and ITCZ regions in tropics. The scatterplot of cloud liquid water retrieved from observation versus scattering index (Fig. 3) for a 20-day period further confirms that the scattering effect becomes stronger nearly linearly where CLW becomes larger. Moreover, the scattering index is also helpful to detect strong scattering where CLW is small. Negative scattering index is also noticed in the figure and it can reach $-10.0 \mathrm{~K}$, but the 


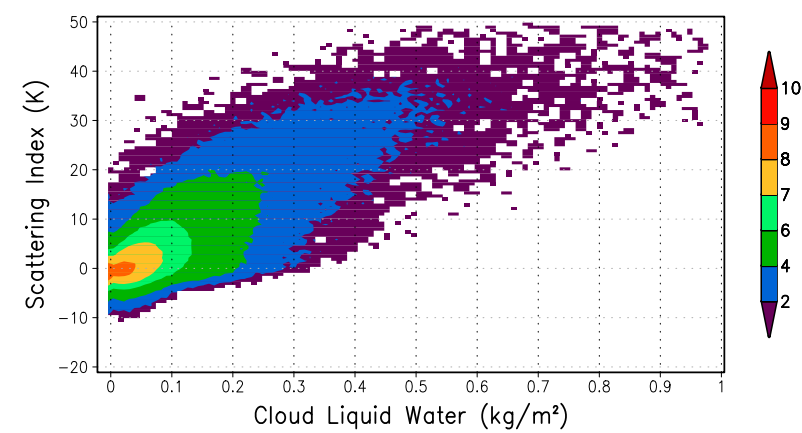

FIG. 3. Scatterplot of cloud liquid water $\left(\mathrm{kg} \mathrm{m}^{-2}\right)$ vs scattering index (K) for ATMS for a 20-day period from 1 to 20 Jun 2015. The color bar shows the common logarithm of data counts.

larger negative SI values only correspond to small CLW values $\left(\mathrm{CLW}<0.1 \mathrm{~kg} \mathrm{~m}^{-2}\right.$ ). The majority of the radiances within $\mathrm{SI} \leq 10.0 \mathrm{~K}$ are associated with CLW $\leq 0.3 \mathrm{~kg} \mathrm{~m}^{-2}$. The impact of the scattering effect on the OmFs of channels $17-22$ is also examined. Figure 4 shows the $\mathrm{OmF}$ bias with respect to observation scattering index for channels 17-22 over ocean. It is seen that channels 17-22 all have negative OmF bias accompanied by positive SI, and the magnitude of $\mathrm{OmF}$ bias becomes larger as the SI increases. Similarly, OmF standard deviation (STDV; Fig. 5) increases with SI value. Among these channels, the scattering has the largest impact on channel 17 and smallest impact on channel 22. Observations from channels 1-7 and 16-22 with $|\mathrm{SI}|>10.0 \mathrm{~K}$ are considered to be affected by strong scattering and excluded in this study. The threshold value of $10.0 \mathrm{~K}$ is chosen empirically.

The final test for quality control is the gross error check. For cloudy AMSU-A radiance assimilation in the operational GFS system, a bias-corrected radiance datum with OmF magnitude larger than 3 times the observation error is excluded from the data assimilation system, where the observation error could be as large as $20.0 \mathrm{~K}$ for cloudy radiances. An approximate $10 \%$ increase in the use of AMSU-A channels 1-5 was observed when moving from the clear-sky approach to the all-sky approach (Zhu et al. 2016). However, because of the strict gross error check used on the ATMS radiances that are assimilated in the clear-sky approach in the current operational system, the data usage of ATMS radiances would increase remarkably if a similar AMSU-A gross error check was applied to ATMS radiances in the all-sky approach. Instead, a biascorrected ATMS radiance with OmF magnitude larger

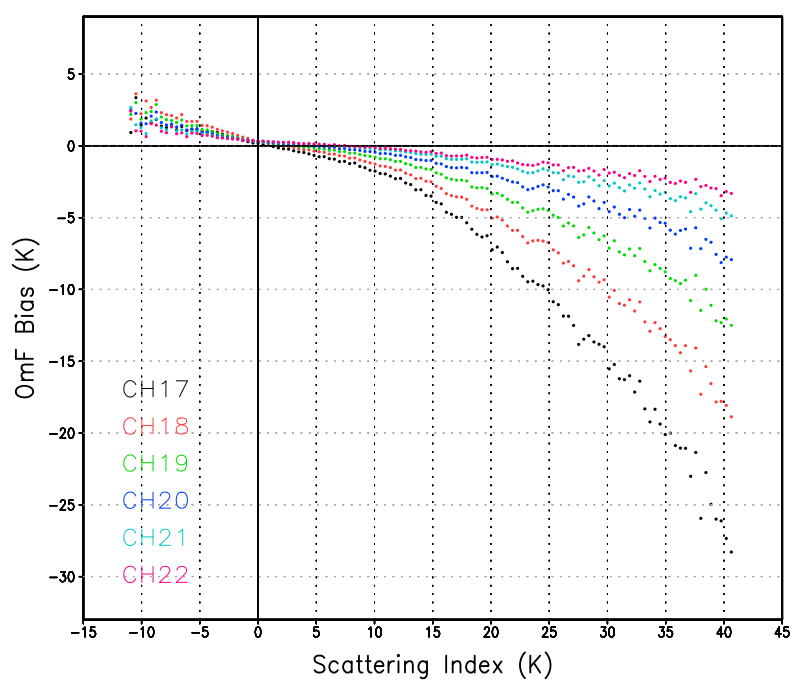

FIG. 4. OmF bias with respect to observation scattering index for channels $17-22$ over ocean.

than a prespecified threshold or 3 times the observation error (see section $3 b$ for more information on observation error), whichever is smaller, is excluded from the data assimilation system. A threshold value of $10.0 \mathrm{~K}$ is applied to ATMS channels $1-6,16$ and $17-$ 22 in the all-sky approach. The threshold value of $10.0 \mathrm{~K}$ is chosen because this value delivers a percentage of data increase for the ATMS channels 1-6 comparable to the corresponding AMSU-A channels, and much larger percentages of data increases for MHSlike channels. More detailed information on ATMS radiance usage can be found in section 5 . The threshold value is expected to become even larger, or removed, when radiances affected by strong scattering are assimilated in the future.

\section{b. Observation error}

Like all-sky AMSU-A, the observation error of ATMS radiance is assigned as a function of the symmetric cloud amount (Geer and Bauer 2011; Geer et al. 2012), following the observed behavior of first guess departures. The symmetric cloud amount used in this study is $\overline{C L W}$, the average of the estimates of CLW over the ocean from either the observation, $C L W_{\text {obs }}$, or the first guess, $\mathrm{CLW}_{\mathrm{fg}}$. The symmetric observation error $e^{o}$ is specified as a function of $\overline{C L W}$ in fitting to the OmF standard deviation.

$$
e^{o}=\left\{\begin{array}{cl}
e_{\mathrm{clr}}^{o} & \left(\overline{\mathrm{CLW}} \leq C_{\mathrm{clr}}\right) \\
e_{\mathrm{clr}}^{o}+\lambda\left(\overline{\mathrm{CLW}}-C_{\mathrm{clr}}\right) & \left(C_{\mathrm{clr}}<\overline{\mathrm{CLW}}<C_{\mathrm{cld}}\right) \quad \text { where } \\
e_{\mathrm{cld}}^{o} & \left(\overline{\mathrm{CLW}} \geq C_{\mathrm{cld}}\right)
\end{array}\right.
$$




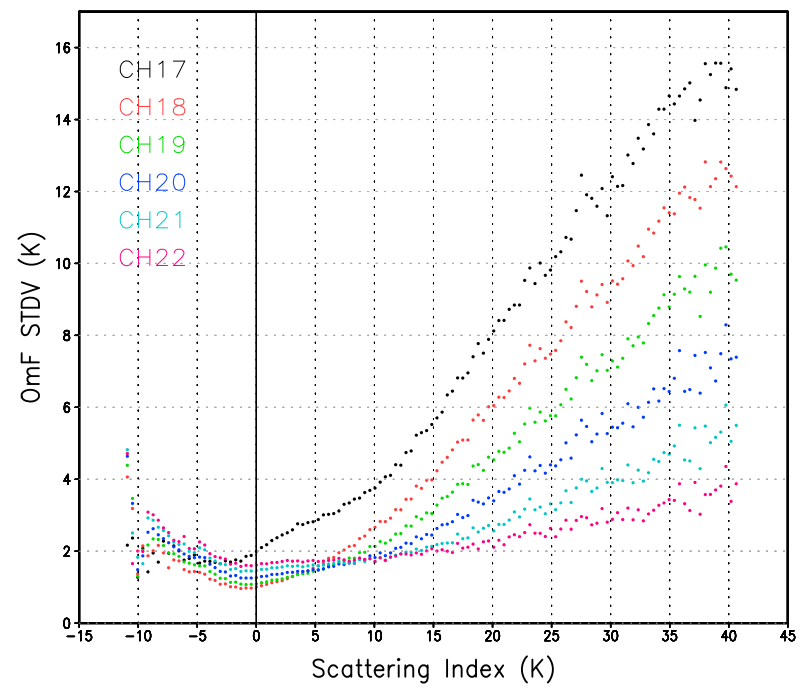

FIG. 5. As in Fig. 4, but for OmF standard deviation.

$$
\lambda=\frac{e_{\mathrm{cld}}^{o}-e_{\mathrm{clr}}^{o}}{C_{\mathrm{cld}}-C_{\mathrm{clr}}}
$$

$C_{\text {clr }}$ and $C_{\text {cld }}$ are the two cloud threshold values for the piecewise linear fitting, which are channel dependent, and $e_{\mathrm{clr}}^{o}$ and $e_{\mathrm{cld}}^{o}$ are the observation errors for radiances associated with clear-sky radiances and $\overline{\mathrm{CLW}} \geq C_{\mathrm{cld}}$, respectively; $e_{\mathrm{clr}}^{o}$ corresponds to the observation errors used in the operational clear-sky ATMS radiance assimilation but the values of $e_{\mathrm{clr}}^{o}$ we use in the all-sky approach are slightly smaller. The parameters used for the ATMS radiances are provided in Table 3. It is known that there are strong correlations among ATMS tropospheric temperature sounding and humidity sounding channels. Although studies on applying correlated observation error have been ongoing at NCEP (Bathmann 2018), the correlations are not modeled in the observation error in the present operational GFS or in this study. Instead, the operational observation error and the symmetric observation error used in this study are both inflated (relative to the OmF standard deviation) to hedge against the lack of observation error correlations.

The same SDOEI procedure as for AMSU-A (Zhu et al. 2016) is also applied to the ATMS radiances. The observation error is inflated based on surface elevation, the sensitivities of brightness temperature to the surface emissivity and skin temperature, and surface-to-space transmittance. Additional inflation is also applied empirically for the all-sky approach using the physically based factors on which it is assumed the observation error (through primarily the CRTM) is dependent. The physically based factors considered include cloud placement difference and cloud liquid
TABLE 3. A list of the parameter values used in the all-sky radiance observation error assignment for ATMS channels 1-7 and 16-22.

\begin{tabular}{ccccc}
\hline \hline Channel & $C_{\text {clr }}\left(\mathrm{kg} \mathrm{m}^{-2}\right)$ & $C_{\text {cld }}\left(\mathrm{kg} \mathrm{m}^{-2}\right)$ & $e_{\text {clr }}^{o}(\mathrm{~K})$ & $e_{\text {cld }}^{o}(\mathrm{~K})$ \\
\hline 1 & 0.03 & 0.35 & 4.50 & 20.00 \\
2 & 0.03 & 0.38 & 4.50 & 25.00 \\
3 & 0.03 & 0.40 & 4.50 & 12.00 \\
4 & 0.02 & 0.45 & 2.50 & 7.00 \\
5 & 0.03 & 0.50 & 0.55 & 3.50 \\
6 & 0.08 & 1.00 & 0.30 & 3.00 \\
7 & 0.15 & 1.00 & 0.30 & 0.80 \\
16 & 0.02 & 0.35 & 4.00 & 19.00 \\
17 & 0.03 & 0.50 & 4.00 & 30.00 \\
18 & 0.03 & 0.50 & 3.50 & 25.00 \\
19 & 0.03 & 0.50 & 3.00 & 16.00 \\
20 & 0.03 & 0.50 & 3.00 & 12.00 \\
21 & 0.05 & 0.50 & 3.00 & 9.00 \\
22 & 0.10 & 0.50 & 3.00 & 6.50 \\
\hline
\end{tabular}

water difference between the first guess and observation, a scattering index equal to or larger than $9 \mathrm{~K}$ (Grody et al. 1999), and the surface wind speed. While the first two are the dominant factors, the inflation based on the latter two factors is very small. Since many useful cloudy radiance observations are associated with large OmFs, situation-dependent observation error inflation allows us to assimilate these observations and retrieve meteorologically important information without shocking the system.

\section{c. Bias correction}

For satellite radiance data, observation bias may arise from the instrument, calibration processes, and forward radiative transfer model. Biases can be much larger than signals. With the unbiased observation assumption in the formulation of our data assimilation system, it is important to remove the bias from radiance data before assimilating them. Furthermore, bias correction and quality control closely interact with each other in the GSI. On one hand, quality control is applied to bias-corrected radiance data; on the other hand, radiance bias correction is performed with the quality-controlled radiance data, anchored on the observations that are not bias corrected. Any poor handling of the two processes will degrade the analysis and may even make the system settle at an incorrect solution.

Following the all-sky AMSU-A, the ATMS radiances that pass the quality control procedures are bias corrected in the GSI's variational bias correction framework (Derber and Wu 1998; Zhu et al. 2014a,b) but using a selected sample. The purpose of sample selection is to avoid the impact of model errors, especially the temporal-spatial and phase errors of hydrometeors, on the radiance data bias correction, while 

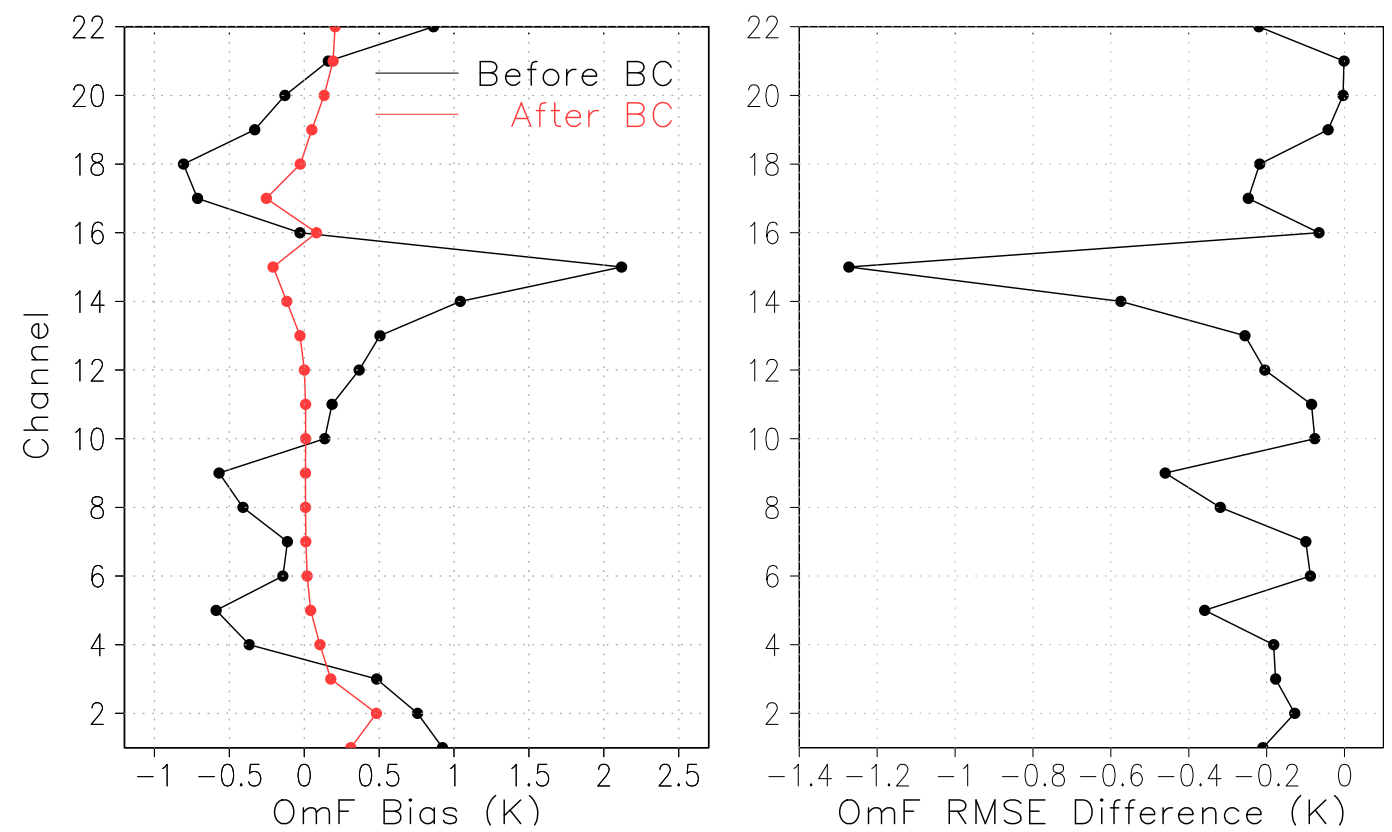

FIG. 6. (left) The ATMS radiance OmF bias before (black line) and after (red line) bias correction and (right) the reduction of the OmF RMSE for the period from 1 to 20 Jun 2015.

preserving useful cloud information from the $\mathrm{OmF}$ with mismatched cloud information. As in Zhu et al. (2016), the data sample where clouds are either present or absent in both the observation and first guess are used to derive the predictor coefficients. Radiance data

$$
\tilde{h}(\mathbf{x}, \delta \boldsymbol{\beta})=\left\{\begin{array}{cl}
h(\mathbf{x})+\sum_{k=1}^{N} \beta_{b k} p_{k}(\mathbf{x}) & \text { (if with mismatched cloud, over ocean) } \\
h(\mathbf{x})+\sum_{k=1}^{N} \beta_{b k} p_{k}(\mathbf{x})+\sum_{k=1}^{N} \delta \beta_{k} p_{k}(\mathbf{x}) & \text { (otherwise) }
\end{array}\right.
$$

that have mismatched cloud information from the first guess, where observation is clear-sky but first guess is cloudy or vice visa, are bias corrected using the latest bias coefficients available. Thus, the observation operator $\tilde{h}$ of the ATMS radiance data can be written as where $\mathbf{x}$ is the model state or GSI control vector, and $h(\mathbf{x})$ represents the radiative transfer model. Letting $\beta_{b k}$ denote the latest available estimate of the predictor coefficient at each outer loop and $\delta \beta_{k}$ the coefficient increment, the total bias is written as a linear combination of a set of predictors $p_{k}(\mathbf{x}), k=$ $1,2, \ldots, N$, and $p_{1}=1 . \delta \boldsymbol{\beta}$ is also a GSI control vector, and is updated along with $\mathbf{x}$ in the GSI minimization procedure.

In the all-sky approach, the bias predictors for ATMS include global offset, the lapse rate convolved with the channel's weighting function, the square of the lapse rate convolved with the channel's weighting function, emissivity sensitivity, and zenith angle bias correction terms. The CLW bias predictor, which is used in the clear-sky approach to remove the cloud effect, is no longer needed. The all-sky ATMS radiance OmFs are examined before and after bias correction for the period from 1 to 20 June 2015, and the results show that the bias correction works well for ATMS radiances. After bias correction, the ATMS radiance OmF bias (Fig. 6, left) is improved across all channels, and the difference of RMSE between OmFs with and without bias correction (Fig. 6, right) also indicates a slight reduction of OmF RMSE.

\section{First guess departure comparison and data consistency}

In current data assimilation systems, there is an increasingly large number of observations available. Special attention should be paid to not only the accuracy and stability of the observations from each data source, but also to the discrepancies caused by 

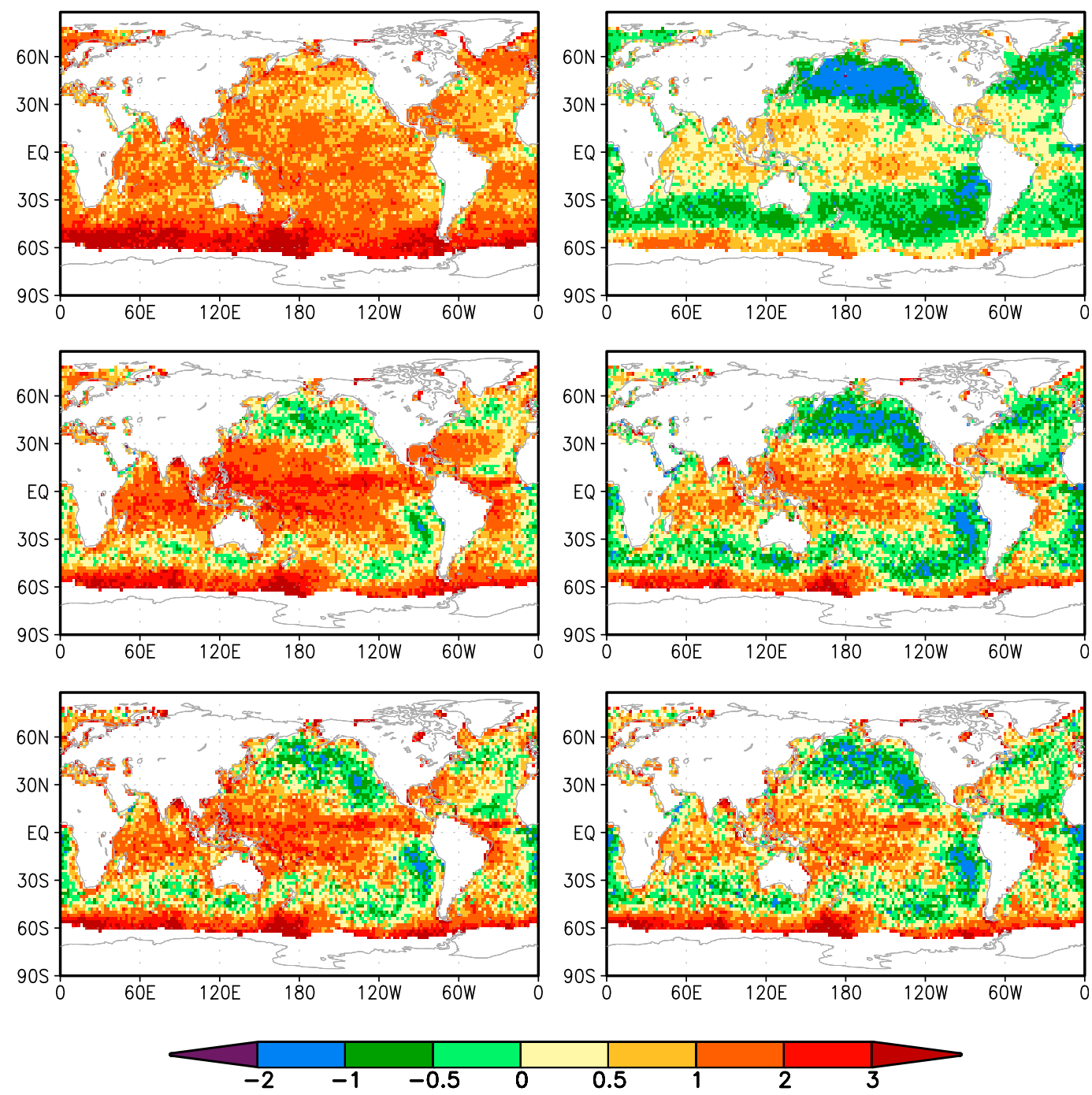

FIG. 7. One-month averaged OmF over water of June 2015: (left) before and (right) after bias correction for ATMS channel 1 in the (top) clear-sky approach, (middle) ATMS, and (bottom) AMSU-A NOAA 19 channel 1 in the all-sky approach. The unit is K.

different processing procedures. As conflicting observational information will degrade the analysis quality and system performance, it is necessary to assess consistency among different data sources. In the current operational GFS, radiance data from several microwave sensors are assimilated differently. The AMSU-A radiances over ocean are assimilated using the all-sky approach operationally, but both ATMS and MHS radiances are assimilated using the clear-sky approach. Only perfectly clear-sky radiances are assimilated for MHS, while both clear-sky radiances and radiances affected by only thin clouds are used for ATMS, with this thin-cloud effect removed by a CLW bias predictor. In this study, when ATMS radiances over water are assimilated in the all-sky approach, the CLW bias predictor is removed as cloud information is accounted for explicitly in the calculation of simulated brightness temperature, and ATMS radiances affected by thick clouds are also used. In this section, to gain a better understanding of our usage of radiance data, the patterns of used ATMS radiance OmFs over water are examined in both clear-sky and all-sky approaches, and compared with those of AMSU-A and MHS radiances. Radiances over land, which are assimilated using the clearsky approach in the GSI, are not plotted in Figs. 7 and 8.

The averaged OmFs over water of June 2015 for used radiances of ATMS channel 1 in the clear-sky and all-sky approaches are presented in the top and middle 

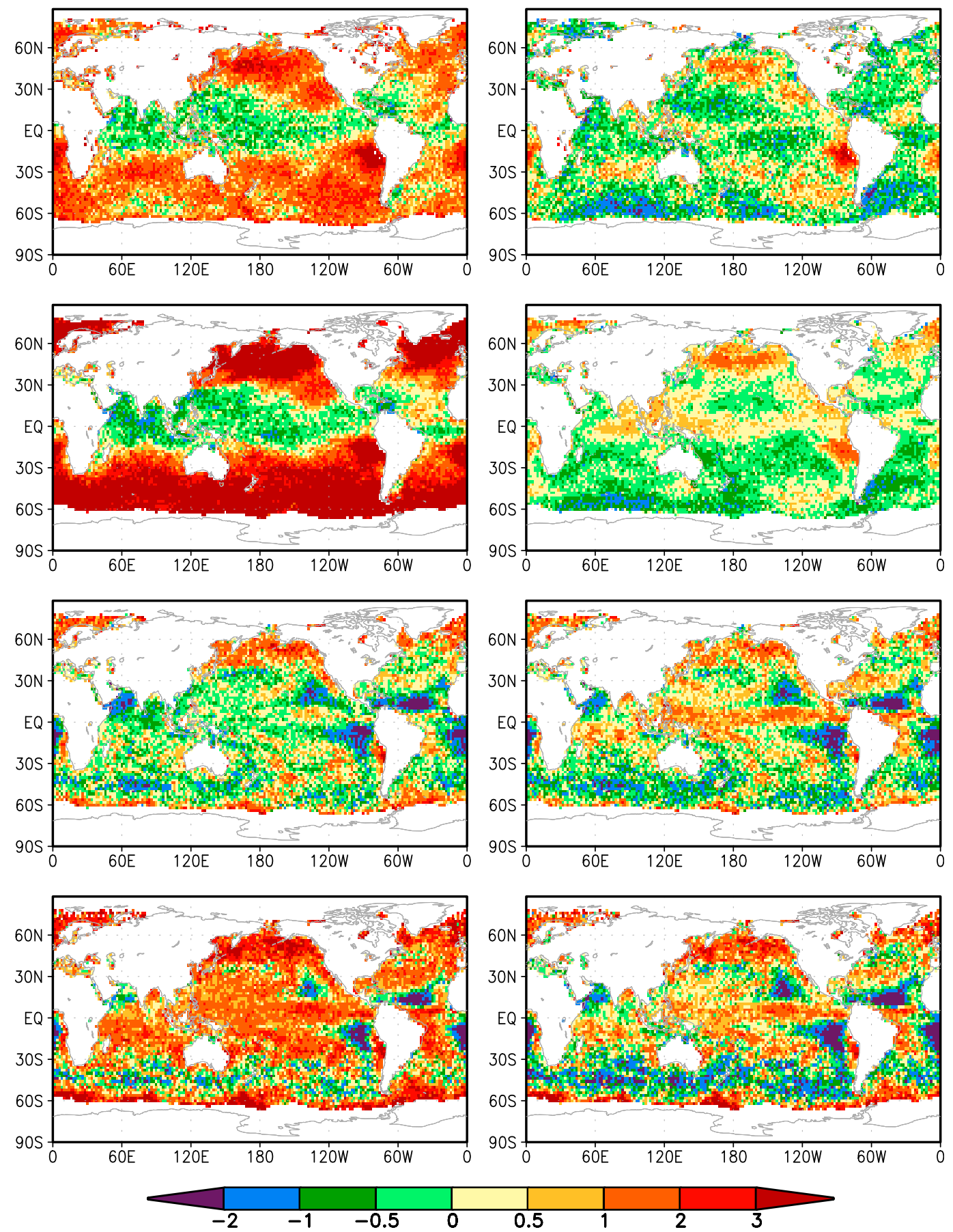

FIG. 8. One-month averaged OmF over water of June 2015: (left) before and (right) after bias correction for (top row) MHS channel 1 and (second row) ATMS channel 16 in the clear-sky approach, and (third row) ATMS channel 16 and (bottom row) AMSU-A channel 15 in the all-sky approach. The unit is K.

row, respectively, of Fig. 7, and compared with AMSU-A NOAA-19 channel 1 in the all-sky approach (bottom row). The left column is for the OmFs before bias correction and the right column is for after bias correction. It is seen that for ATMS channel 1, although there are significant pattern differences in the OmF before bias correction between the clear-sky and all-sky approach, similar patterns are observed after bias correction. This similarity indicates that the cloud liquid water bias predictor used in the clear-sky approach 
and other standard bias predictors work well for channel 1. It also appears that the bias correction procedure is doing less work in the all-sky case, which is generally accepted as a good thing. More importantly, when comparing all-sky ATMS and AMSU-A channel 1, consistent OmF patterns are observed before and after bias correction.

However, inconsistencies are noticed between the clear-sky and the all-sky approaches for ATMS channel 16 (Fig. 8). ATMS channel 16 and its closest matches, MHS channel 1 and AMSU-A channel 15 are presented in this figure. Comparing the $\mathrm{OmF}$ patterns before (left column) and after (right column) bias correction, bias correction apparently has a big impact on these channels as well. After bias correction, while the OmF patterns for ATMS channel 16 (row 2) and MHS channel 1 (row 1) are alike in the clear-sky approach, they are significantly different in several regions from the OmF patterns of ATMS channel 16 (row 3) and AMSU-A channel 15 (row 4) in the all-sky approach. One of such regions is to the west of the South American continent. Unlike the consistent negative OmFs of ATMS and AMSU-A channel 1 (Fig. 7) in this region from both the clearsky and all-sky approaches, ATMS channel 16 and MHS channel 1 exhibit positive OmFs in the clear-sky approach, while ATMS channel 16 and AMSU-A channel 15 show negative OmFs in the all-sky approach. One possible cause for the ATMS channel 16 OmF difference between the two approaches may lie in the inadequacy of the CLW bias predictor in the clear-sky approach. Since this bias predictor is calculated using the brightness temperatures of channels 1 and 2 (Grody et al. 2001), where it seems to be working well, it may not be adequate for channel 16 . The transition from the clear-sky approach to the all-sky approach makes ATMS radiances more consistent among their own low-peaking channels and with the all-sky AMSU-A radiances. As for MHS radiances, since the quality control procedures are designed to only allow clear-sky radiances to be assimilated in the clear-sky approach, the positive bias-corrected OmFs west of the South American continent may suggest that some cloudy MHS radiance observations leak through the quality control and get used in the GSI. Therefore, stricter quality control may be needed for MHS radiances in the clear-sky approach, or it may be needed to move to the all-sky framework for MHS.

Another clear pattern shown in both all-sky AMSU-A and ATMS OmFs after bias correction is the positive OmFs of channels 1 and 16 in the tropics. A similar feature is also observed in channels $2-4$. In the current
TABLE 4. Data counts of assimilated radiances from ATMS channels in experiments CTL and allskyATMS during the period from 21 May to $31 \mathrm{Jul} 2015$. The parentheses indicate that the radiances of the channel are not actively assimilated but monitored, and the values in parentheses are the data counts that are used in the passive channel bias correction procedure.

\begin{tabular}{cccc}
\hline \hline Channel & CTL & allskyATMS & Increase $(\%)$ \\
\hline 1 & 1538368 & 1709385 & 11.1 \\
2 & 1551877 & 1681362 & 8.3 \\
3 & 1548907 & 1714885 & 10.7 \\
4 & 1551877 & 1724424 & 11.1 \\
5 & 1550754 & 1724654 & 11.2 \\
6 & 1551762 & 1724654 & 11.1 \\
7 & 2375273 & 2195506 & -7.6 \\
8 & 2905571 & 2915386 & 0.3 \\
9 & 2902434 & 2912206 & 0.3 \\
10 & 2905773 & 2915549 & 0.3 \\
11 & 2905615 & 2915324 & 0.3 \\
12 & 2902834 & 2912669 & 0.3 \\
13 & 2853550 & 2864869 & 0.4 \\
14 & 2869216 & 2880735 & 0.4 \\
15 & $(2882540)$ & $(2892713)$ & $(0.4)$ \\
16 & 1512752 & 1625523 & 7.5 \\
17 & 1226179 & 1691378 & 37.9 \\
18 & 1367395 & 1702719 & 24.5 \\
19 & 1340310 & 1705903 & 27.3 \\
20 & 1300993 & 1704664 & 31.0 \\
21 & 1264378 & 1702137 & 34.6 \\
22 & 1209882 & 1700043 & 40.5 \\
\hline
\end{tabular}

GFS forecast model, since subgrid-scale clouds and precipitation are not available in the forecast model output, only grid-scale clouds are used in the radiance simulation calculation for Figs. 7 and 8. The lack of model clouds is evident in the simulated brightness temperature calculation at the lower and middle levels in the ITCZ and SPCZ regions. An effort is under way at the NCEP to take into account the subgrid-scale clouds and precipitation in the all-sky radiance assimilation.

\section{Impact on forecast skills}

Two-and-a-half-month-long cycled T670/T254 low-resolution data assimilation experiments are performed with the GFS system from 1800 UTC 14 May to 31 July 2018. The control run (CTL) uses all observations used in the operational GFS system. Like the operational GFS, only AMSU-A radiances over water are used with the all-sky approach in the CTL, while other radiances including ATMS radiances are assimilated with the clear-sky approach. The all-sky ATMS experiment (allskyATMS) is different from the CTL in that ATMS radiances over water are assimilated with the allsky approach. Because of the system spinup, the first 
TABLE 5. Comparison of experiments CTL and allskyATMS on data counts and OmF RMSE (the unit is $\mathrm{K}$ ) of assimilated radiances from AMSU-A NOAA-19 cloud-sensitive channels during the period from 21 May to 31 Jul 2015.

\begin{tabular}{cccccc}
\hline \hline & \multicolumn{2}{c}{ Data counts } & & \multicolumn{2}{c}{ OmF RMSE } \\
\cline { 2 - 3 } \cline { 5 - 6 } Channel & CTL & allskyATMS & & CTL & allskyATMS \\
\hline 1 & 1626713 & 1631442 & & 3.26 & 3.27 \\
2 & 1628273 & 1633174 & & 4.06 & 4.06 \\
3 & 1633475 & 1638213 & & 2.67 & 2.65 \\
4 & 1634196 & 1638927 & & 1.34 & 1.34 \\
5 & 1634331 & 1639047 & & 1.17 & 1.17 \\
15 & 1627441 & 1633403 & & 4.95 & 4.95 \\
\hline
\end{tabular}

week of experimental results are excluded from the statistical calculations used in this study.

Overall, it is seen from Table 4 that more ATMS radiances are assimilated in the allskyATMS experiment than in experiment CTL using the operational clearsky approach. The increases of assimilated radiances range from $7.5 \%$ to $11.2 \%$ for channels $1-6$ and 16 , and $24.5-40.5 \%$ for channels $17-22$. The majority of these increases are due to the assimilation of the radiances affected by thick clouds and the relaxation of the gross error check. The application of AAPP spatial averaging to channels $17-22$ in the all-sky approach also has a small contribution to their data increases. A small percentage of increase is also observed in the high-peaking channels $8-15$. The only exception is channel 7; its data count is reduced by $7.6 \%$ because of the newly added interchannel quality control and scattering effect screening in the all-sky approach.

In the operational GFS system, a large amount of AMSUA radiances (from $N O A A-15, N O A A-18, N O A A-19$, Aqua, MetOp-A, and MetOp-B) are assimilated in the all-sky approach. As part of AMSU-A NOAA-19 data coverage overlaps with ATMS, the data count and OmF RMSE of the assimilated radiances from AMSU-A NOAA-19 cloud-sensitive channels are examined and summarized in Table 5. While there is little change in OmF RMSE between experiments CTL and allskyATMS, slightly more AMSU-A radiances are assimilated in experiment allskyATMS because of the use of ATMS radiances in the all-sky approach. This is in agreement with the results presented in section 4 that the cloudy ATMS introduction is consistent with the existing use of AMSU-A in the all-sky approach. Similar results are obtained for the AMSU-A radiances from other satellites, but with smaller increase in data counts.

The impact of ATMS radiance assimilation in the all-sky approach on the fits to the rawinsonde data is also assessed. Figure 9 displays the comparisons of
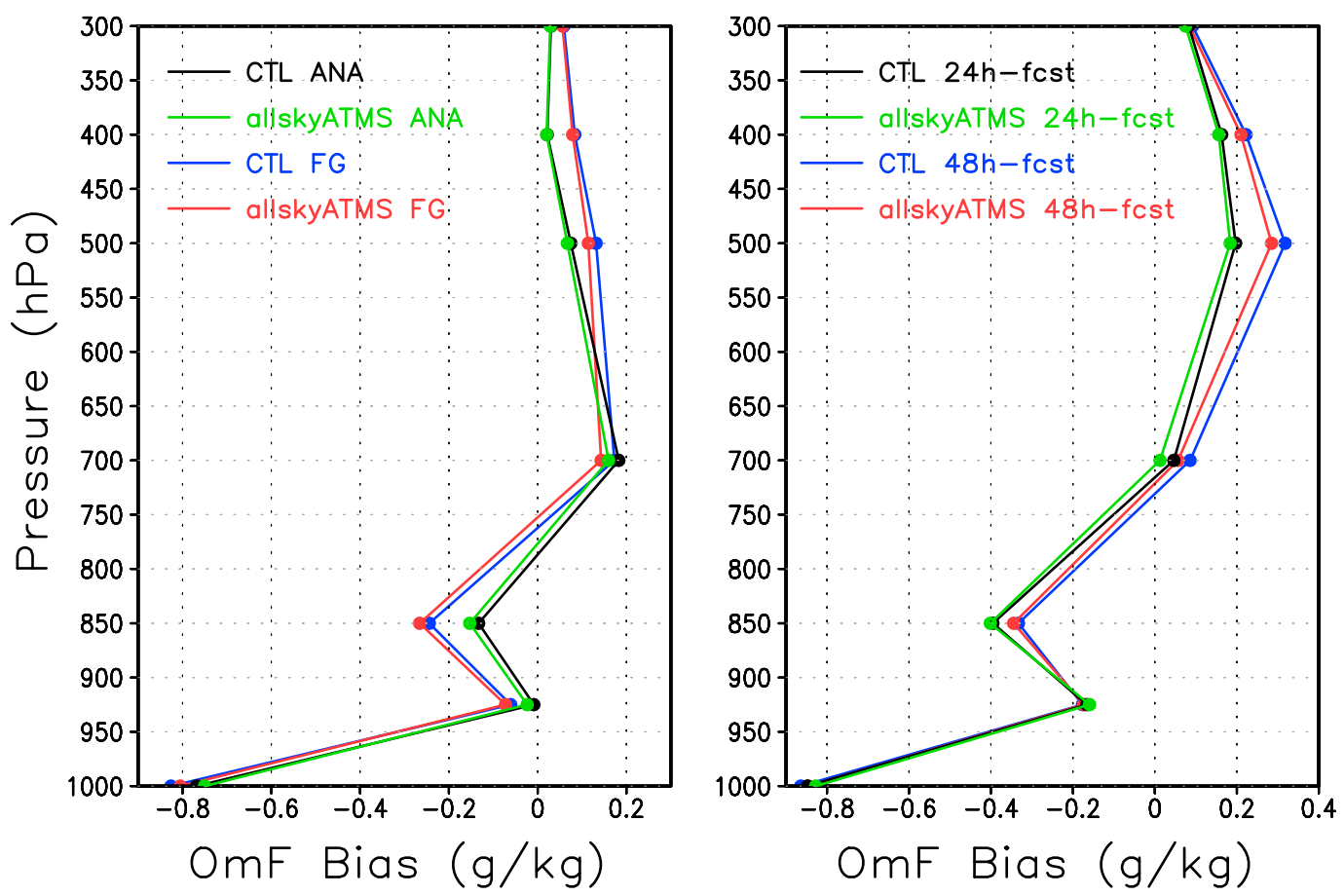

FIG. 9. The bias comparison of fits to rawinsonde specific humidity observations $\left(\mathrm{g} \mathrm{kg}^{-1}\right)$ between (left) experiment CTL and allskyATMS: analysis (ANA) and first guess (FG) fits, (right) 24- and 48-h forecast (fcst) fits during the period from 21 May to 31 Jul 2015. 

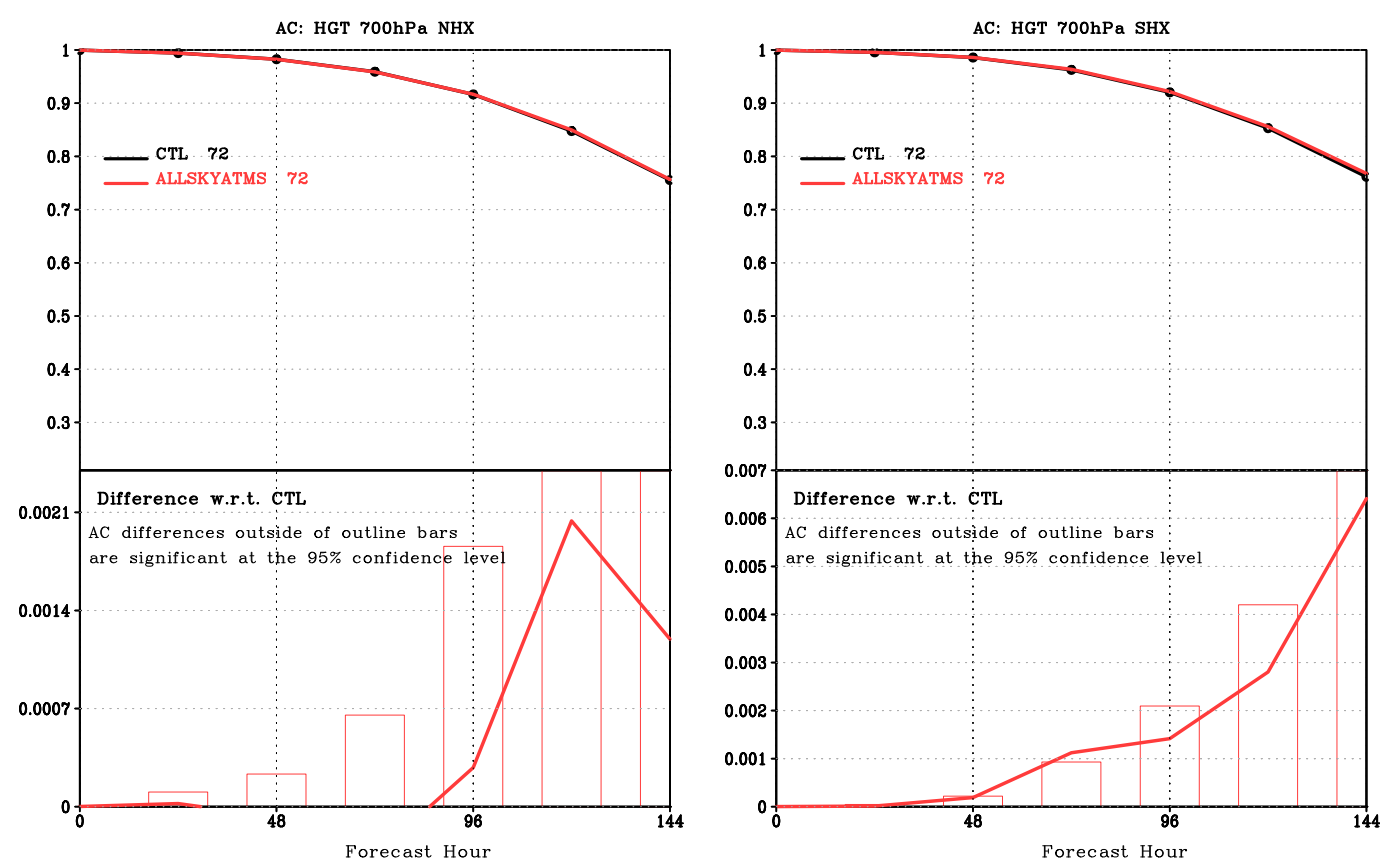

FIG. 10. (top) Geopotential height anomaly correlation (AC) at $700 \mathrm{hPa}$ for the (left) Northern and (right) Southern Hemispheres during the period from 21 May to 31 Jul 2015. (bottom) The difference of AC between the allskyATMS and CTL.

analysis, first guess, 24- and 48-h forecast fits to rawinsonde specific humidity observations between the experiments CTL and allskyATMS in the tropics. The biases of analysis and first guess fits are slightly degraded below $800 \mathrm{hPa}$ but improved above $800 \mathrm{hPa}$, and the improvement is persistent at 24and 48 -h forecasts. However, no noticeable changes are observed in Northern and Southern Hemispheres and in the fits to rawinsonde temperature and wind data.

In terms of the forecast skill, the assimilation of cloudy ATMS radiances is seen to have a neutral impact on the anomaly correlation of geopotential height at $700 \mathrm{hPa}$ (Fig. 10) in the Northern Hemisphere (left panel) and slightly positive impact in the Southern Hemisphere (right panel). Similar behavior at $500 \mathrm{hPa}$ is also observed. For temperature anomaly correlation, small positive impact is seen at day 3 in the Southern Hemisphere, and the results at $850 \mathrm{hPa}$ are presented in Fig. 11. As for RMSE of vector wind, mixed results are observed in the Northern Hemisphere and tropics. The difference of the RMSE between the allskyATMS and CTL for the Southern Hemisphere is shown in the right panel of Fig. 12. The green color indicates RMSE reduction in the allskyATMS experiment, but the improvement is very small and typically not statistically significant. The RMSE reduction is significant at the $95 \%$ confidence level only at $500 \mathrm{hPa}$ on days 2 and 3 (Fig. 13) and from 850 to $400 \mathrm{hPa}$ only on day 3 (figures not shown).

Overall, the improvement on the forecast skill is very small, but our 4D EnVar GFS system is able to make use of all the dynamical and physical information (including hydrometeor evolution) in the ensemble and infer temperature, humidity, and wind information from satellite radiances through the background error covariance. Although in the configuration of the operational 4D EnVar GFS system, the ensemble is not updated at the GSI outer loops to reflect the changes of model states, and the usage of AMSU-A radiances overwhelms ATMS SNPP radiances, the beneficial generalized tracer effect (Geer et al. 2018) due to ATMS radiances assimilated in the all-sky approach is observed.

\section{Conclusions and future work}

In this study, following the all-sky radiance assimilation framework done for the AMSU-A radiances in the operational GFS system, we have expanded the all-sky approach to ATMS radiance assimilation and water vapor channels. To facilitate quality control and observation error assignment procedures, a common beamwidth is taken for all ATMS channels and the surface properties are calculated based on 


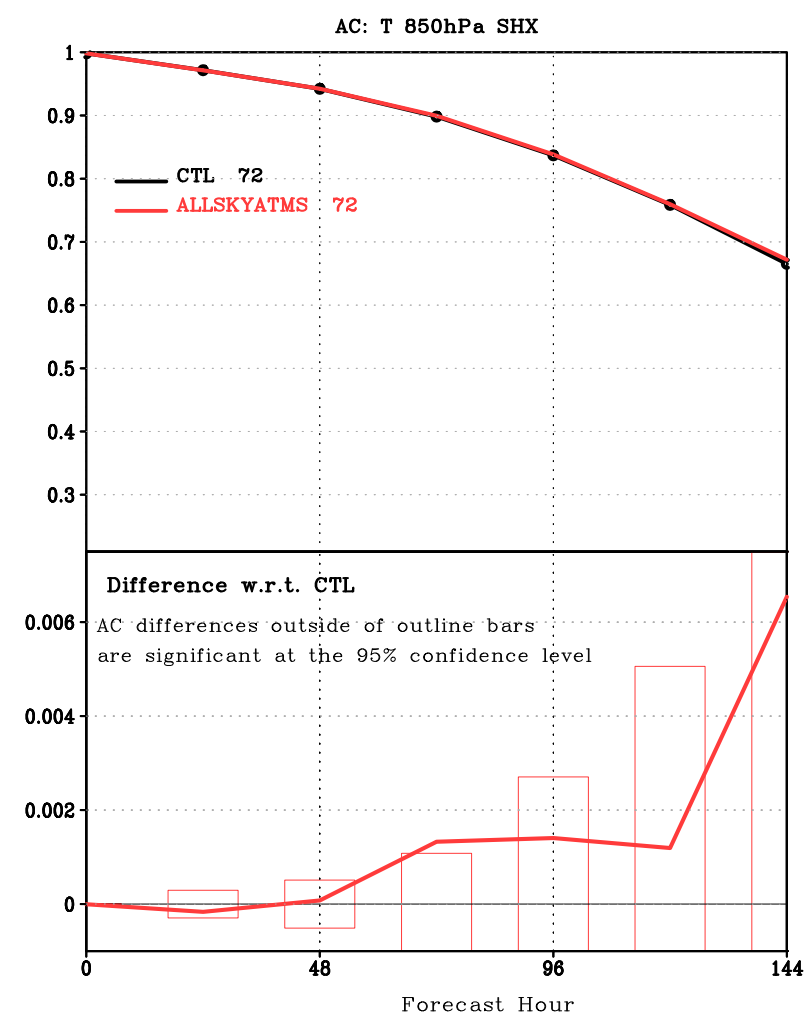

FIG. 11. (top) Temperature anomaly correlation (AC) at $850 \mathrm{hPa}$ for the Southern Hemisphere and (bottom) the difference of AC between the allskyATMS and CTL during the period from 21 May to $31 \mathrm{Jul}$ 2015. CTL.

the FOV size and shape. Additional quality control procedures have been constructed to handle the radiances with large OmF around coastlines and cryosphere boundaries and radiances affected by strong scattering with the introduction of MHS-like channels of higher frequencies into the all-sky framework. This study helps to shed light on the effectiveness of our radiance data usage in the operational GFS system. With the comparisons of monthly averaged OmFs among AMSU-A, ATMS and MHS, the conflicting OmF patterns among these sensors are identified and possible causes are discussed. The effort on expanding the all-sky approach to ATMS radiances has made the use of radiance data in the GSI more consistent among various sensors. As a small improvement in the fits to rawinsonde specific humidity data is found to persist in the 48-h forecast, the inclusion of cloudy ATMS radiances is found to have an overall neutral impact on the model forecast skills, with small improvement in the Southern Hemisphere mainly at day 3 . This all-sky ATMS radiance work has been included in the real-time parallel of the FiniteVolume Cubed Sphere Dynamical Core (FV3) GFS beta version for the operational implementation in 2019. With the inclusion of more ATMS radiances from $N O A A-20$ in this implementation, the generalized tracer effect due to cloudy ATMS radiances is expected to be more evident.

One of the remaining research topics is to account for the non-Gaussian distribution of the all-sky radiance innovation data explicitly. The ATMS radiance OmFs histograms for cloud-sensitive channels exhibit obvious non-Gaussian features with a fat tail. Tentative explorations have been conducted (but not presented in this paper) to see whether the treatment of the pronounced non-Gaussian distribution can be advantageously enhanced using, in addition to the existing situation-dependent observation error inflation, an adaptation of variational quality control (VQC) formulated based on a superlogistic distribution (Purser 2011, 2018). The new probability model of this VQC is constructed by convolving a parameterized "chevron function" with the normalized logistic function. Compared with the original VQC formulation (Purser 1984; Lorenc and Hammon 1988; Andersson and Järvinen 1998; Bauer et al. 2010) being a linear combination of Gaussian and flat distributions, which has been applied to only conventional data in the GSI, the new VQC formulation greatly reduces the possibility of multiple-minima in the cost function. The impact of the mathematically formulated VQC on the observation weight is examined against the physically based SDOEI. As expected, the VQC and SDOEI have distinct and potentially complementary characteristics. The SDOEI handles the radiances that have large cloud discrepancies between the observations and first guess, while the VQC merely reduces the weight of observation that are at the tails of the data distribution. While many cloudy locations tend to have large OmFs because of the inaccuracies of model physical parameterization schemes and the CRTM, the largest differences between the VQC weightreduction factor and the SDOEI in our experiments are noticed in the tropics. In our two-and-a-halfmonth-long cycled data assimilation experiments, no obvious benefit on the forecast skills is observed with the application of the VQC, presumably because presently the large gross errors involved are more in the model's misrepresented cloud fields and not in the measurements themselves. It is therefore decided that the VQC is not included for this implementation. More future research will continue on this topic since some non-Gaussian gross errors in representation are expected to arise from the cloudy radiance forward operator, even given an essentially correct model 

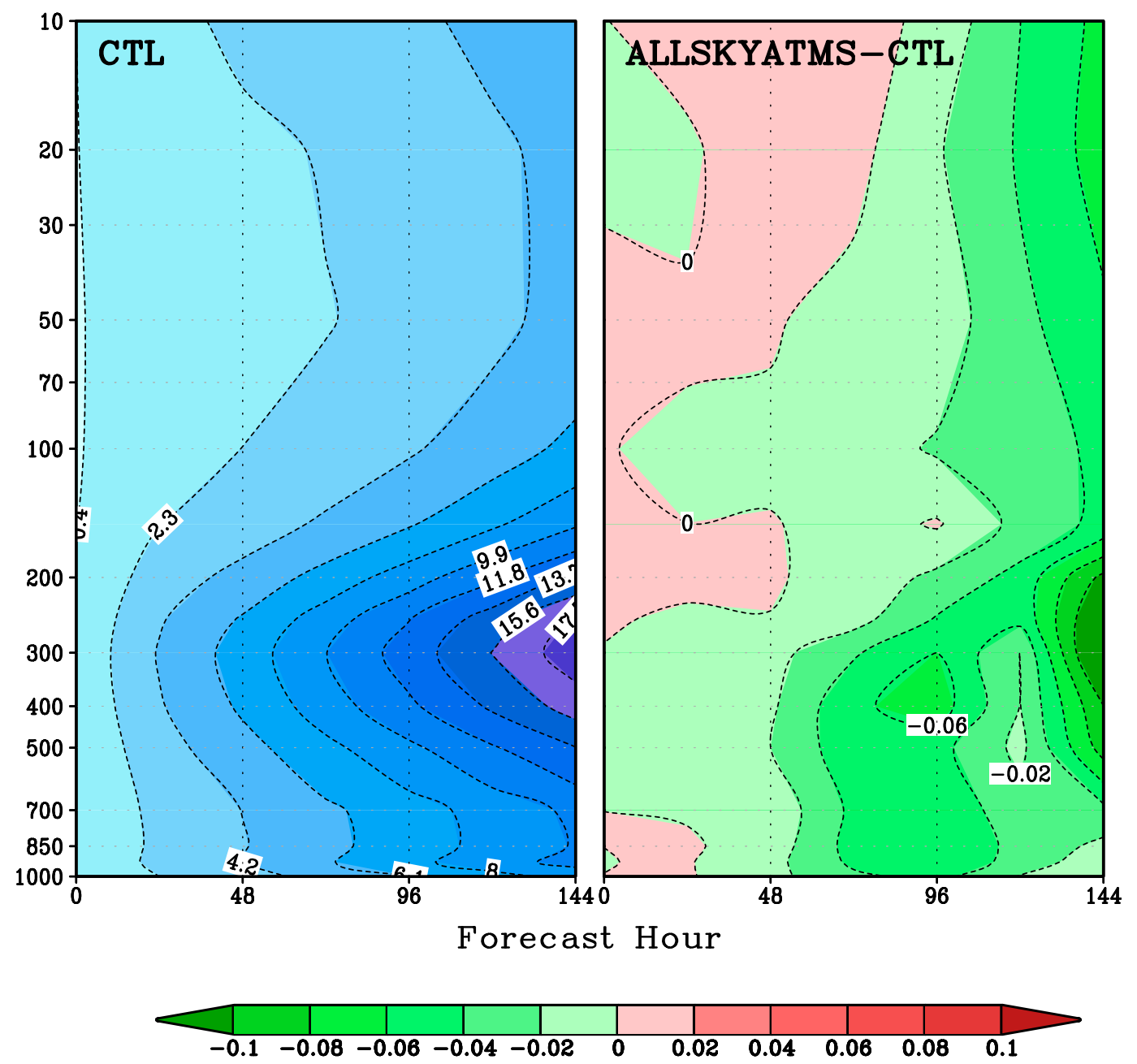

FIG. 12. (left) The RMSE results of wind vector forecast in Southern Hemisphere for the CTL and (right) the corresponding RMSE difference between the allskyATMS and CTL during the period from 21 May to 31 Jul 2015. The RMSE is computed against each system's own analysis, and the unit is $\mathrm{m} \mathrm{s}^{-1}$.

cloud field; therefore, an appropriately tuned VQC scheme should be able to further improve these cloudy radiance assimilations.

As the forecast models are transitioning to the FV3 model with more advanced physics at NCEP, the effort on adapting the all-sky radiance assimilation in the FV3 framework is under way. The all-sky radiance assimilation with individual hydrometeors as cloud control variables will be tested, as cloud liquid water, cloud ice, snow, rain, and graupel become the prognostic variables in the forecast model. In particular, with the newly improved scattering coefficients in the CRTM (Stegmann et al. 2018), the impact of radiances affected by strong scattering will be assessed. More research should also be conducted on other choices of the cloud control variables. Although the current cloud control variable works well, we believe investigation of alternative cloud control variables in the future will be beneficial, especially to help in retaining observational information in the model forecast.

Meanwhile, one current ongoing effort is to account for the subgrid-scale clouds and precipitation in the all-sky radiance assimilation. The lack of clouds and precipitation in the tropics is clearly shown in the monthly averaged ATMS and AMSU-A OmFs. The impact of allowing grid- and subgrid-scale clouds and precipitation to have different optical properties is worthy of exploration in future studies. As further refinements of all-sky assimilation continue, along with the enhancements of the CRTM with improved optical properties, the all-sky radiance assimilation should be expanded to additional instruments and radiances over land. 


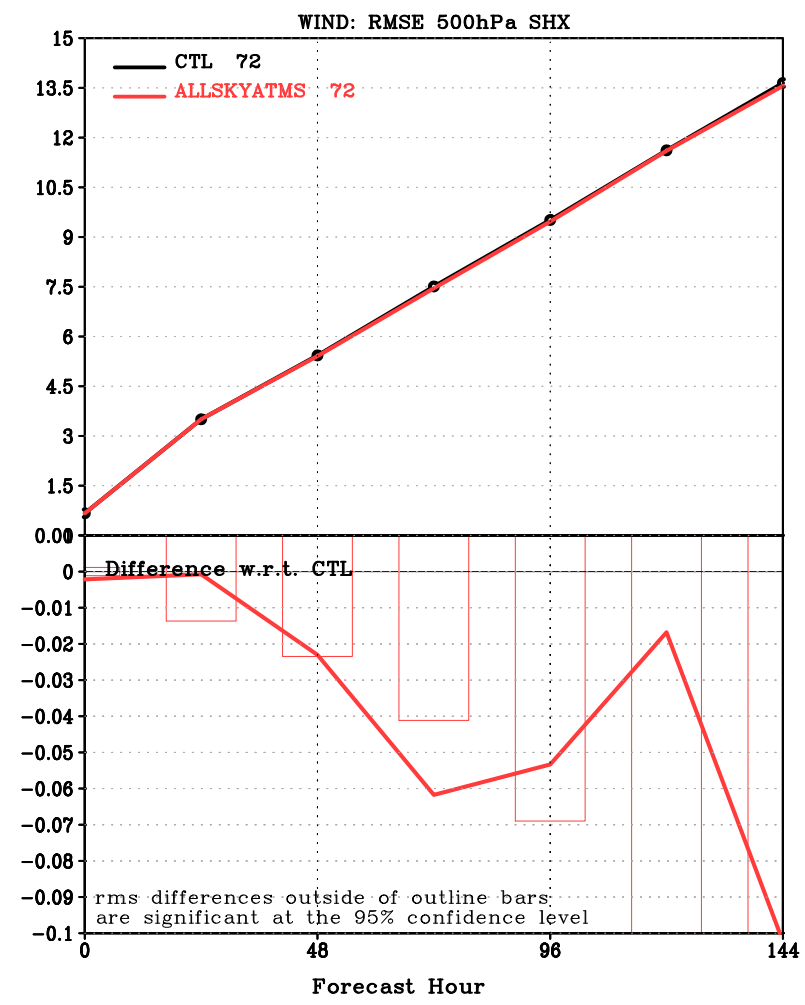

FIG. 13. (top) The wind vector forecast RMSEs at $500 \mathrm{hPa}$ in the Southern Hemisphere for the allskyATMS and CTL experiments, and (bottom) the RMSE difference of allskyATMS with respect to CTL. The RMSE is computed against each system's own analysis, and the unit is $\mathrm{ms}^{-1}$.

Acknowledgments. We thank our EMC colleagues K. Bathmann, A. Collard, J. Derber, D. Groff, H. Liu, R. Mahajan, and D. Parrish for their helpful comments. We also would like to thank the four anonymous reviewers for their helpful comments and suggestions.

\section{REFERENCES}

Andersson, E., and H. Järvinen, 1998: Variational quality control. ECMWF Tech. Memo. 250, 31 pp., https://doi.org/10.21957/ lqz2wn16g.

Baordo, F., and A. Geer, 2015: All-sky assimilation of SSMIS humidity sounding channels over land within the ECMWF system. EUMETSAT/ECMWF Research Rep. 38, 26 pp.

Bathmann, K., 2018: Justification for estimating observation-error covariances with the Desroziers diagnostic. Quart. J. Roy. Meteor. Soc., 144, 1965-1974, https://doi.org/10.1002/qj.3395.

Bauer, P., E. Moreau, F. Chevallier, and U. O'Keeffe, 2006: Multiple-scattering microwave radiative transfer for data assimilation applications. Quart. J. Roy. Meteor. Soc., 132, 1259-1281, https://doi.org/10.1256/qj.05.153.

_, A. J. Geer, P. Lopez, and D. Salmond, 2010: Direct 4D-Var assimilation of all-sky radiances. Part I: Implementation. Quart. J. Roy. Meteor. Soc., 136, 1868-1885, https://doi.org/ 10.1002/qj.659.

Bennartz, R., A. Thoss, A. Dybbroe, and B. Michelson, 2002: Precipitation analysis using the Advanced Microwave
Sounding Unit in support of nowcasting applications. Meteor. Appl., 9, 177-189, https://doi.org/10.1017/S1350482702002037.

Collard, A., J. Derber, R. Treadon, N. Atkinson, J. Jung, and K. Garrett, 2012: Toward assimilation of CrIS and ATMS in the NCEP Global Model. 18th Int. TOVS Study Conf., Toulouse, France, International TOVS Working Group, 1.18, https://cimss.ssec.wisc.edu/itwg/itsc/itsc18/ program/files/Collard_ITSC18.pdf.

Derber, J. C., and W.-S. Wu, 1998: The use of TOVS cloud-cleared radiances in the NCEP SSI analysis system. Mon. Wea. Rev., 126, 2287-2299, https://doi.org/10.1175/1520-0493(1998) 126<2287:TUOTCC $>2.0$.CO;2.

— D. F. Parrish, and S. J. Lord, 1991: The new global operational analysis system at the National Meteorological Center. Wea. Forecasting, 6, 538-547, https://doi.org/10.1175/ 1520-0434(1991)006<0538:TNGOAS > 2.0.CO;2.

Ding, S., P. Yang, F. Weng, Q. Liu, Y. Han, P. v. Delst, J. Li, and B. Baum, 2011: Validation of the community radiative transfer model. J. Quant. Spectrosc. Radiat. Transfer, 112, 1050-1064, https://doi.org/10.1016/j.jqsrt.2010.11.009.

Geer, A. J., and P. Bauer, 2011: Observation errors in all-sky data assimilation. Quart. J. Roy. Meteor. Soc., 137, 2024-2037, https://doi.org/10.1002/qj.830.

,-- , and S. English, 2012: Assimilating AMSU-A temperature sounding channels in the presence of cloud and precipitation. ECMWF Tech. Memo. 670, 41 pp.

— , and Coauthors, 2018: All-sky satellite data assimilation at operational weather forecasting centres. Quart. J. Roy. Meteor. Soc., 144, 1191-1217, https://doi.org/10.1002/qj.3202.

Grody, N., F. Weng, and R. Ferraro, 1999: Application of AMSU for obtaining water vapor, cloud liquid water, precipitation, snow cover and sea ice concentration. 10th Int. TOVS Study Conf., Boulder, CO, International TOVS Working Group, 230-240, http://library.ssec.wisc.edu/research_Resources/ publications/pdfs/ITSC10/grody01_ITSC10_1999.pdf.

—, J. Zhao, R. Ferraro, F. Weng, and R. Boers, 2001: Determination of precipitable water and cloud liquid water over oceans from the NOAA 15 advanced microwave sounding unit. J. Geophys. Res., 106, 2943-2953, https://doi.org/10.1029/ 2000JD900616.

Kleist, D. T., and K. Ide, 2015: An OSSE-based evaluation of hybrid variational-ensemble data assimilation for the NCEP GFS. Part II: 4D EnVar and hybrid variants. Mon. Wea. Rev., 143, 452-470, https://doi.org/10.1175/MWR-D-13-00350.1.

Liu, Q., and F. Weng, 2006: Advanced doubling-adding method for radiative transfer in planetary atmosphere. J. Atmos. Sci., 63, 3459-3465, https://doi.org/10.1175/JAS3808.1.

Lorenc, A. C., and O. Hammon, 1988: Objective quality control of observations using Bayesian methods. Theory and a practical implementation. Quart. J. Roy. Meteor. Soc., 114, 515-543, https://doi.org/10.1002/qj.49711448012.

Martinet, P., N. Fourrie, V. Guidard, F. Rabier, T. Montmerle, and P. Brunel, 2013: Towards the use of microphysical variables for the assimilation of cloud affected infrared radiances. Quart. J. Roy. Meteor. Soc., 139, 1402-1416, https://doi.org/10.1002/qj.2046.

Migliorini, S., A. Lorenc, and W. Bell, 2017: A moistureincrementing operator for the assimilation of humidity and cloud-sensitive observations: Formulation and preliminary results. Quart. J. Roy. Meteor. Soc., 144, 443-457, https://doi.org/10.1002/qj.3216.

Okamoto, K., 2013: Assimilation of overcast cloudy infrared radiances of the geostationary MTSAT-1R imager. Quart. 
J. Roy. Meteor. Soc., 139, 715-730, https://doi.org/10.1002/ qj.1994.

Parrish, D. F., and J. C. Derber, 1992: The National Meteorological Center's spectral statistical interpolation analysis system. Mon. Wea. Rev., 120, 1747-1763, https://doi.org/ 10.1175/1520-0493(1992)120<1747:TNMCSS > 2.0.CO;2.

Purser, R. J., 1984: A new approach to the optimal assimilation of meteorological data by iterative Bayesian analysis. 10th Conf. on Weather Forecasting and Analysis, Clearwater Beach, FL, Amer. Meteor. Soc., 102-105.

2011: Mathematical principles of the construction and characterization of a parameterized family of Gaussian mixture distributions suitable to serve as models for the probability distributions of measurement errors in nonlinear quality control. NOAA/NCEP Office Note 468, 42 pp.

_ 2018: Convenient parameterization of super-logistic probability models of effective observation error. NOAA/NCEP Office Note 495, 8 pp., https://doi.org/10.25923/kvmz-vf34.

Stegmann, P. G., G. Tang, P. Yang, and B. T. Johnson, 2018: A stochastic model for density-dependent microwave snow- and graupel scattering coefficients of the NOAA JCSDA community radiative transfer model. J. Quant. Spectrosc. Radiat. Transfer, 211, 9-24, https://doi.org/10.1016/j.jqsrt.2018.02.026.

Wang, X., and T. Lei, 2014: GSI-based four-dimensional ensemble-variational (4DEnsVar) data assimilation: Formulation and single-resolution experiments with real data for NCEP Global Forecast System. Mon. Wea. Rev., 142, 3303-3325, https://doi.org/10.1175/MWR-D-13-00303.1.
Weng, F., L. Zhao, R. R. Ferraro, G. Poe, X. Li, and N. C. Grody, 2003: Advanced microwave sounding unit cloud and precipitation algorithms. Radio Sci., 38, 8086-8096, https://doi.org/ 10.1029/2002RS002679.

Whitaker, J., and T. M. Hamill, 2002: Ensemble data assimilation without perturbed observations. Mon. Wea. Rev., 130, 1913-1924, https:// doi.org/10.1175/1520-0493(2002)130<1913:EDAWPO>2.0.CO;2.

Wu, W.-S., R. J. Purser, and D. F. Parrish, 2002: Three-dimensional variational analysis with spatially inhomogeneous covariances. Mon. Wea. Rev., 130, 2905-2916, https://doi.org/10.1175/15200493(2002)130<2905:TDVAWS >2.0.CO;2.

Zhao, Q., and F. H. Carr, 1997: A prognostic cloud scheme for operational NWP models. Mon. Wea. Rev., 125, 19311953, https://doi.org/10.1175/1520-0493(1997)125<1931: APCSFO $>2.0 . \mathrm{CO} ; 2$.

Zhu, Y., J. Derber, A. Collard, D. Dee, R. Treadon, G. Gayno, and J. A. Jung, 2014a: Enhanced radiance bias correction in the National Centers for Environmental Prediction's Gridpoint Statistical Interpolation data assimilation system. Quart. J. Roy. Meteor. Soc., 140, 1479-1492, https://doi.org/10.1002/qj.2233.

— , and Coauthors, 2014b: Variational bias correction in the NCEP's data assimilation system. 19th Int. TOVS Study Conf., Jeju Island, South Korea, International TOVS Working Group, http://cimss.ssec.wisc.edu/itwg/itsc/itsc19/program/papers/ 10_02_zhu.pdf.

— , and Coauthors, 2016: All-sky microwave radiance assimilation in NCEP's GSI analysis system. Mon. Wea. Rev., 144, 4709-4735, https://doi.org/10.1175/MWR-D-15-0445.1. 